\title{
Pre-immigration Screening for Tuberculosis in South Korea: A Comparison of Smear- and Culture-Based Protocols
}

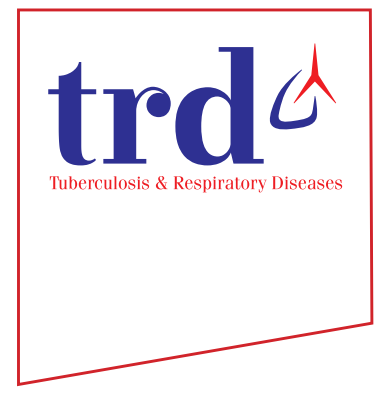

\author{
Sangyoon Lee, M.D., Ph.D. ${ }^{1(\mathbb{D})}$, Ji Young Ryu, M.D., M.Sc. ${ }^{2}$ and Dae-Hwan Kim, M.D., Ph.D. ${ }^{2}$ (i) \\ ${ }^{1}$ Department of Occupational and Environmental Medicine, Inha University Hospital, Incheon, ${ }^{2}$ Department of Occupational \\ and Environmental Medicine, Inje University Haeundae Paik Hospital, Busan, Korea
}

Background: Tuberculosis (TB) is the most important disease screened for upon patient history review during preimmigration medical examinations as performed in South Korea in prospective immigrants to certain Western countries. In 2007, the U.S. Centers for Disease Control and Prevention (CDC) changed the TB screening protocol from a smearbased test to the complete Culture and Directly Observed Therapy Tuberculosis Technical Instructions (CDOT TB TI) for reducing the incidence of TB in foreign-born immigrants.

Methods: This study evaluated the effect of the revised (as compared with the old) protocol in South Korea.

Results: Of the 40,558 visa applicants, 365 exhibited chest radiographic results suggestive of active or inactive TB, and 351 underwent sputum tests (acid-fast bacilli smear and Mycobacterium tuberculosis culture). To this end, using the CDOT TB TI, 36 subjects (88.8 per $10^{5}$ of the population) were found to have TB, compared with only seven using the older U.S. CDC technical instruction (TI) $(\mathrm{p}<0.001)$. In addition, there were six drug-resistant cases which were identified ( 16.7 per $10^{5}$ of the population), two of whom had multidrug-resistance (5.6 per $10^{5}$ of the population).

Conclusion: The culture-based 2007 TI identified a great deal of TB cases current to the individuals tested, as compared to older U.S. CDC TI.

Keywords: Tuberculosis; Immigrants; Screening; Republic of Korea

\section{Introduction}

Some countries, including the United States, Canada, and Australia, require that prospective visitors seeking immigra-

\footnotetext{
Address for correspondence: Dae-Hwan Kim, M.D., Ph.D.

Department of Occupational and Environmental Medicine, Inje

University Haeundae Paik Hospital, 875 Haeun-daero, Haeundae-gu, Busan 48108, Korea

Phone: 82-51-797-0369, Fax: 82-51-797-0313

E-mail: kimdh@paik.ac.kr

Received: Jan. 16, 2018

Revised: Apr. 5, 2018

Accepted: Jul. 2, 2018

Published online: Sep. 28, 2018

(c) It is identical to the Creative Commons Attribution Non-Commercial License (http://creativecommons.org/licenses/by-nc/4.0/).
}

tion, marriage, study, employment, working-holiday, or other visas must undergo a medical examination in a designated hospital. Identification of active tuberculosis (TB) cases is vital because the incidence thereof is much higher in South Korea (86.0 per $10^{5}$ of the population) than in the United States (3.1 per $10^{5}$ of the population), Canada (5.2 per $10^{5}$ of the population), and Australia (6.4 per $10^{5}$ of the population) in $2014^{1}$.

In 1991, the U.S. Centers for Disease Control and Prevention (CDC) drafted a technical instruction (TI) for TB screening and treatment of visa applicants; this TI featured chest radiography (CXR) and three consecutive acid-fast bacilli (AFB) smear tests on sputum if the CXR results were suggestive of TB. Although since 1991, the U.S. CDC TI for overseas TB screening has stipulated three consecutive AFB smear tests after an abnormal CXR, transmission of TB from AFB smearnegative patients remains important because of the relatively low predictive value of these tests. In 2007, the U.S. CDC expanded the TI to include three consecutive Mycobacterium tuberculosis (MTB) cultures of sputum in addition to the smear tests and mandated drug susceptibility testing (DST) 
and directly observed therapy (DOT) for those undergoing TB treatment (Culture and Directly Observed Therapy [CDOT] TB TI). Although some differences in the age of the subjects exist, the visa programs of many countries, including Canada, Australia, New Zealand, and the United States, follow the revised TI of the U.S. CDC.

The prevalence of smear- and/or culture-positive TB has diminished noticeably according to the nationwide Korean TB surveys that were conducted every 5 years from 1965 (940 per $10^{5}$ of the population) to 1995 (219 per $10^{5}$ of the population) $)^{2}$. The Korean Tuberculosis Surveillance System (KTBS), an internet-based system of case notification, was adopted in 2000 , but this system has been criticized. Notification of TB cases, especially by private institutions, may be neglected or incomplete, which could lead to an underestimation of the TB burden $^{3}$

We compared the incidence of TB among visa applicants screened using the culture-based protocol with that determined using the smear-based protocol. Additionally, we indirectly estimated the status of TB in South Korea from the number of cases of active TB identified in medical screening examinations of visa applicants.

\section{Materials and Methods}

\section{Subjects and study design}

A total of 43,488 visa applicants underwent medical examinations in a private general hospital in Busan from May 1, 2011, to July 31, 2015 at the request of the embassies of the United States, Canada, and Australia. Prior to 2007, overseas

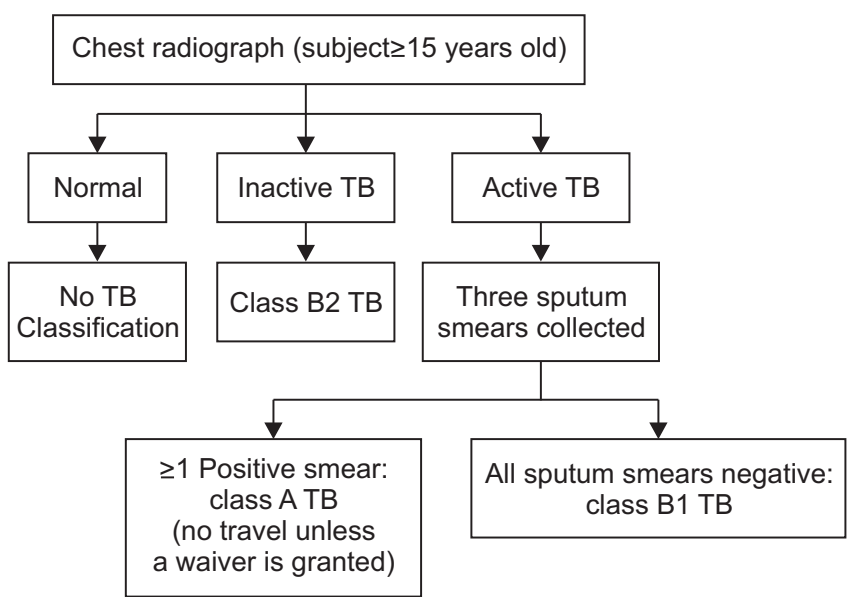

Figure 1. The U.S. Centers for Disease Control and Prevention 1991 technical instructions for pre-immigration TB screening and treatment. Class A TB: active TB disease; Class B1 TB: chest radiographic data consistent with active TB; Class B2 TB: chest radiographic data consistent with inactive TB; TB: tuberculosis.
TB screening of pre-immigrants 15 or more years of age before departure for the U.S. comprised CXR. If the CXR results were abnormal, which is suggestive of active TB, three consecutive AFB smear tests were performed on sputum. If any of the tests were positive, the subject was considered to have class A TB. If all smear tests were negative, the subject was classified with class B1 TB (Figure 1). The TI was expanded in 2007; three consecutive AFB sputum tests are still required, in addition to MTB culture tests if the CXR results are abnormal, in subjects with evident signs or symptoms of TB on medical examination, any history of TB, or human immunodeficiency virus infection. If any of the sputum or culture tests are positive, the subject is considered to have class A TB. If all smear and culture tests are negative, the subjected is classified with class B1 TB. Class A patients then undergo DST and DOT ${ }^{4}$ (Figure 2). We retrospectively compared the number of class A TB patients among visa applicants using the (enhanced) 2007 TI and the $1991 \mathrm{TI}$.

\section{Laboratory examinations}

The laboratory examination followed the TB diagnostic standards of the U.S. CDC and the American Thoracic Society ${ }^{5}$. Three consecutive AFB smear tests on sputum and three MTB cultures were performed on three successive days; the

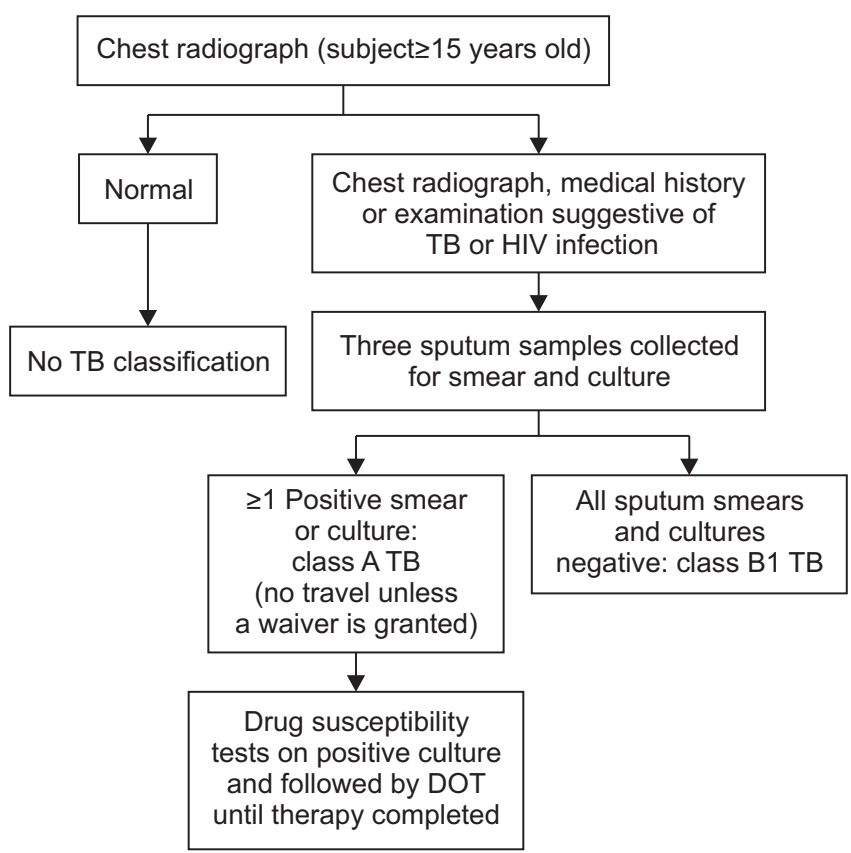

Figure 2. The complete Culture and Directly Observed Therapy Tuberculosis Technical Instructions of U.S. Centers for Disease Control and Prevention. Class A TB: active TB disease; Class B1 TB: chest radiographic data consistent with active TB; TB: tuberculosis; HIV: human immunodeficiency virus; DOT: directly observed therapy. 
examiner donned protective gloves and a N95 mask when collecting sputum. The room in which sputum was collected was under negative pressure and was fitted with a ultravioletbased sterilizing system ${ }^{6}$. If sputum production was difficult, sputum was induced by inhalation of an aerosol consisting of sterile hypertonic saline. Sputum specimens were stained using the Ziehl-Neelsen method and cultured on both solid medium (3\% egg-based Ogawa medium) and in liquid medium (Middlebrook 7H12 medium); subjects with a single positive result on $\mathrm{AFB}$ smear or culture tests were considered TB positive. Detection of non-TB mycobacteria (NTM) was not considered a positive result. DST was performed using the absolute concentration method in Lowenstein-Jensen medium. Pyrazinamide susceptibility testing was performed by the pyrazinamidase test. If a culture test was positive, DST was performed for amikacin, capreomycin, cycloserine, ethambutol, isonazid, kanamycin, levofloxacin, moxifloxacin, ofloxacin, para-aminosalicylic acid, prothionamide, pyrazinamide, rifabutin, rifampicin, and streptomycin. Multidrug-resistant (MDR) TB (MDR-TB) was defined as TB resistant to both isoniazid and rifampicin, while extensive drug-resistant TB (XDR-TB) was defined TB that was also resistant to any fluoroquinolone (ofloxacin, moxifloxacin, or levofloxacin) and at least one second-line injectable drug (amikacin, capreomycin, or kanamycin), as well as $\mathrm{MDR}^{7}$.

\section{Statistical analysis}

We documented the general characteristics of the population and calculated the rate of TB cases. The chi-square test and Fisher's exact test were used to compare proportions. Continuous variables are expressed as the mean \pm standard deviations (SDs) and categorical variables were expressed as numbers and percentages. All tests of significance were twosided, and a p-value of $<0.05$ was deemed to reflect statistical significance. All analyses were performed using SAS software version 9.2 (SAS Institute Inc., Cary, NC, USA).

\section{Ethics statement}

An ethics review was performed, and permission to collect data from the visa applicants was obtained from the institutional review board of Haeundae Paik Hospital (IRB No. 201607-009-001). The requirement for informed consent was waived because this study involved only a review of the medical records and posed minimal risk to the subjects.

\section{Results}

Among the 43,488 applicants, we excluded those under 15 years of age $(n=444)$ who did not take CXR according to the U.S. CDC protocol, as well as foreigners $(\mathrm{n}=592)$. Applicants who visited several times because of repeat visits to one country or visits to several countries $(n=1,887)$ were counted only once. Finally, we evaluated 40,558 subjects. The average age $( \pm \mathrm{SD})$ of the population was $26.8( \pm 6.6)$ years; $21,980(54.2 \%)$ were aged $25-34$ years, 15,532 (38.3\%) were $15-24$ years, with those of younger age (15-34 years) constituting $90 \%$ of the population. Thirty-one applicants stated that they had previously undergone treatment for TB. Abnormal CXR results suggestive of either inactive or active TB were obtained from 365 subjects $\left(0.9 \%, 899.9\right.$ per $10^{5}$ of the population) (Table 1$)$.

Fourteen subjects refused further sputum examinations, and 351 underwent three sputum AFB smear tests and MTB cultures. Seven subjects had at least one positive smear test and 33 at least one positive MTB culture. Twenty-nine subjects were negative on the AFB smear tests but positive on the culture tests. One positive sputum test was associated with a positive MTB culture in 11 cases, two sputum tests were associated with positive cultures in 17 cases, and all three sputum tests were positive in five cases, together with positive culture data (Table 2).

TB was identified in 36 subjects (88.8 per 105 of the population) using the CDOT TB TI but in only seven using the 1991 U.S. CDC TI ( $\mathrm{p}<0.001)$. When evaluated by sex, 22 males $(96.8$ per $10^{5}$ of the population) and 14 females (78.5 per $10^{5}$ of the population) were classified with TB using the $2007 \mathrm{TI}$, but only four males and three females were classified with TB using the $1991 \mathrm{TI}$; these differences were statistically significant

Table 1. General characteristics of the study population $(n=40,558)$

\begin{tabular}{|lc|}
\hline \multicolumn{1}{|c|}{ Characteristic } & No. (\%) \\
\hline Sex & $22,718(56.0)$ \\
\hline Male & $17,840(44.0)$ \\
\hline Female & \\
\hline Uniting country & $1,406(3.5)$ \\
\hline Canada & $10,691(26.4)$ \\
\hline Australia & $28,461(70.2)$ \\
\hline Age, mean \pm SD $(\mathrm{yr})$ & $26.8 \pm 6.6$ \\
\hline $15-24$ & $15,532(38.3)$ \\
\hline $25-34$ & $21,980(54.2)$ \\
\hline $35-44$ & $1,855(4.6)$ \\
\hline $45-54$ & $739(1.8)$ \\
\hline $55-64$ & $281(0.7)$ \\
$\geq 65$ & $171(0.4)$ \\
\hline Previous treatment history of TB & $31(0.1)$ \\
\hline Suggestive of TB on CXR & $365(0.9)$ \\
\hline
\end{tabular}

TB: tuberculosis; CXR: chest X-ray. 
$(\mathrm{p}<0.001$ and $\mathrm{p}=0.007$, respectively). Six cases were resistant to at least one TB drug (16.7\%), and two were MDR-TB (5.6\%) according to the CDOT TB TI (Table 3 ).

Table 2. Sputum test results of those with abnormal chest radiographs $(\mathrm{n}=351)$

\begin{tabular}{|lc|}
\hline \multicolumn{1}{|c|}{ Variable } & No. (\%) \\
\hline AFB smear and MTB culture & $315(89.7)$ \\
\hline AFB smear $(-)$ and MTB culture $(-)$ & $3(0.9)$ \\
\hline AFB smear $(+)$ and MTB culture $(-)$ & $29(8.3)$ \\
\hline AFB smear $(-)$ and MTB culture $(+)$ & $4(1.1)$ \\
\hline AFB smear $(+)$ and MTB culture $(+)$ & $7(2.0)$ \\
\hline No. of positive AFB smears & 5 \\
\hline One & 0 \\
\hline Two & 2 \\
\hline Three & $33(9.4)$ \\
\hline No. of positive MTB cultures & 11 \\
\hline One & 17 \\
\hline Two & 5 \\
\hline Three & \\
\hline
\end{tabular}

AFB: acid-fast bacilli; MTB: Mycobacterium tuberculosis.

\section{Discussion}

In total, 36 cases of TB were detected in visa applicants using the CDOT TB TI ( 88.8 per $10^{5}$ of the population). The incidence was found to be already high in younger subjects (118.3 per $10^{5}$ of the population aged 25-34 years) and to increase with age. When the older smear-based U.S. CDC TI was used, only seven applicants were classified as having TB; thus, 29 positive TB cases $(80.6 \%)$ were missed when the older TI was used.

The prevalence of TB in South Korea has decreased with an improving economy, but TB control remains unsatisfactory ${ }^{8}$. A public health problem is evident. The incidence of $\mathrm{TB}$ (86 per $10^{5}$ of the population) is the highest among the 34 countries of the Organization for Economic Cooperation and Development (OECD) in 2014; the average TB incidence in the other 33 OECD countries was only 9.76 per $10^{5}$ of the population in $2014^{1}$. In the United States from 2001 to 2008, South Koreans accounted for $8.1 \%$ of all estimated TB cases among newly arrived students/exchange visitors and for $6.2 \%$ of TB cases among newly arrived tourists and business travelers, ranking as the fourth and seventh most significant sources of infection, respectively ${ }^{9}$.

Immigration has been an important cause of TB in high-income countries. In the United States, for example, $64 \%$ of new TB cases in 2013 were attributable to infections spread from foreign-born subjects ${ }^{10}$. The U.S. CDC conducted a prelimi-

Table 3. Characteristics of the TB cases identified using different technical instructions

\begin{tabular}{|c|c|c|c|c|c|}
\hline \multirow[b]{2}{*}{ Characteristic } & \multicolumn{2}{|c|}{$1991 \mathrm{TI}$} & \multicolumn{2}{|c|}{2007 TI using CDOT } & \multirow[b]{2}{*}{ p-value } \\
\hline & No. (\%) & $\begin{array}{l}\text { Cases per } 10^{5} \text { of } \\
\text { the population }\end{array}$ & No. (\%) & $\begin{array}{l}\text { Cases per } 10^{5} \text { of } \\
\text { the population }\end{array}$ & \\
\hline TB cases identified & $7(17.3)$ & & $36(88.8)$ & & $<0.001$ \\
\hline \multicolumn{6}{|l|}{ Sex } \\
\hline Male & $4(57.1)$ & 17.6 & $22(61.1)$ & 96.8 & $<0.001$ \\
\hline Female & $3(42.9)$ & 16.8 & $14(38.9)$ & 78.5 & 0.007 \\
\hline \multicolumn{6}{|l|}{ Age, yr } \\
\hline $15-24$ & - & - & $3(8.3)$ & 19.3 & 0.250 \\
\hline $25-34$ & $5(71.4)$ & 22.7 & $25(69.4)$ & 113.7 & $<0.001$ \\
\hline $35-44$ & - & - & $3(8.3)$ & 161.7 & 0.499 \\
\hline $45-54$ & $1(14.3)$ & 135.3 & $3(8.3)$ & 406.0 & 0.624 \\
\hline $55-64$ & - & - & $1(2.8)$ & 355.9 & $>0.999$ \\
\hline$\geq 65$ & $1(14.3)$ & 584.8 & $1(2.8)$ & 584.8 & $>0.999$ \\
\hline Drug-resistant TB & & & $6(16.7)^{*}$ & & \\
\hline MDR-TB & - & - & $2(5.6)^{*}$ & - & - \\
\hline XDR-TB & - & - & $0(0)$ & - & - \\
\hline
\end{tabular}

*Percentage of TB cases.

TB: tuberculosis; TI: technical instruction; CDOT: culture and directly observed therapy; MDR: multidrug-resistant; XDR: extensively drugresistant 
nary study on the effectiveness of pre-immigration TB screening programs in Vietnam among 1,179 U.S.-bound immigrants with abnormal CXRs; positive AFB smear results were detected in $82(7.0 \%)$ immigrants, positive MTB culture results in 183 (15.5\%), and negative smear but positive culture results in $120(10.2 \%)^{11}$. Follow-up evaluation from 1999 to 2005 revealed that $7.0 \%$ of smear-negative TB immigrants were diagnosed with active TB after arrival in the United States ${ }^{12}$. A DNA fingerprinting study performed in the United States showed that at least $17 \%$ of TB patients had been infected by smear-negative patients; the relative TB transmission rate from smear-negative to smear-positive patients was found to be 0.22 (95\% confidence interval, $0.16-0.32)^{13}$.

The AFB smear test has a sensitivity of about $53 \%$. A second specimen increases this to $64.4 \%$ and a third specimen increases this to $66.4 \%-69.4 \%^{14}$. This is because $5,000-10,000$ AFB per microliter sputum sample are required for a positive result $^{15}$, compared to 10-100 AFB per microliter for a sputum culture $^{16}$, which has a sensitivity of $80 \%-85 \%$ and specificity of about $98 \% \%^{17-19}$. The low sensitivity of the AFB smear test is also attributable to differences in staining technique, centrifugation speed, reader experience, and the TB prevalence in the population $(30 \%-80 \%)^{17,20}$. In this study, the culture test identified $33 \mathrm{~TB}$ cases, compared to seven for the AFB smear test.

After implementation of culture-based TB screening in subjects from countries with a high TB burden in 2007, the annual number of TB cases diagnosed within 1 year of arrival among foreign-born subjects decreased steadily from 1,326 to 940 (2008-2012) compared with the 1,424-1,626 (mean 1,504) cases (2002-2007) diagnosed prior to implementation of the new TI. Concomitantly, the incidence of smear-negative culture-positive cases increased from 178 (42.4\%) in 2008 to 629 (59.5\%) in $2012^{21}$. Approximately $60 \%$ of immigrants and refugees with TB had negative smear results but positive culture results, according to the U.S. CDC ${ }^{22}$. The enhanced screening program reduced the occurrence of TB within 6 months of arrival in the United States from 86 of 2,049 subjects (4.2\%) (preintervention; applying the $1991 \mathrm{TI}$ ) to 22 of 1,430 subjects (1.5\%) post-intervention (applying the new $2007 \mathrm{TI})^{23}$. In the present study, 29 of 36 cases (80.5\%) were smear-negative but culture-positive using the $2007 \mathrm{TI}$, whereas only seven were diagnosed with TB using the $1991 \mathrm{TI}$.

Drug-resistant TB was not uncommon in this study. DST is included in the enhanced TI to identify drug-resistant TB cases and to treat MDR-TB. In a four-round Korean study on national drug resistance conducted from 1994 to 2004, the prevalence of anti-TB drug resistance increased steadily from $11.3 \%$ to $12.8 \%$ and that of MDR from $1.6 \%$ to $2.7 \%$ of new cases and $27.5 \%$ to $14.0 \%$ of previously treated cases, respectively ${ }^{24}$. In a previous Korean study, $5.8 \%$ of new cases and $20.1 \%$ of previously treated cases had MDR-TB ${ }^{25}$. Another study in South Korea reported that $6.8 \%$ of total TB cases were MDR-TB, and $12.4 \%$ of these were XDR-TB ${ }^{26}$. In this study, six of the 36 patients (16.7\%) diagnosed with TB were resistant to at least one anti-TB drug, and two (5.6\%) had MDR-TB and it was consistent with previous study.

Host immunity against MTB declines with age $\mathrm{e}^{27}$. We found that the number of TB cases per $10^{5}$ of the population increased with age. A high prevalence of latent $\mathrm{TB}$ and TB re-activation may explain this finding. However, the high incidence of TB even in younger subjects suggests that TB remains infectious and is spreading among the young in South Korea. The incidence of TB in this study was 88.8 per $10^{5}$ of the population, which is lower than the 89.6 per $10^{5}$ of the population in the 2013 KTBS annual report of TB in Korea ${ }^{28}$. The incidence of TB among subjects 25-34 years of age was greater in this work that in the 2013 report ( 113.78 vs. 76.4 per $10^{5}$ of the population 25-29 years of age and 60.4 per $10^{5}$ of the population 30-34 years of age, respectively). The difference between our findings and the KTBS report becomes greater with increasing age, with the exception of those 15-24 years of age (19.3 vs. 37.2 per $10^{5}$ of the population $15-19$ years of age and 62.7 per $10^{5}$ of the population 20-24 years of age, respectively).

Much effort has focused on controlling TB in South Korea. The latent TB infection (LTBI) guidelines are reinforced in the revision of the 2014 Korean guidelines for TB because LTBI treatment is important for reducing the incidence of $\mathrm{TB}^{29}$. Moreover, identification of active TB cases is also important for reducing the prevalence of $\mathrm{TB}$.

This study had several limitations. First, it was performed in a single private hospital and was of a retrospective design, so the results are not representative of all visa applicants in South Korea. A multi-center prospective study would provide a clearer picture. Second, the study population consisted mostly of young subjects; less than $10 \%$ were over 35 years of age, possibly indicating selection bias. Third, AFB smear-positive and MTB culture-negative cases were common due to NTM or contamination of sputum. Fourth, most applicants refused to report their history of $\mathrm{TB}$ treatment to avoid further sputum tests; indeed, 14 applicants with CXR results suggestive of TB refused sputum tests. If these subjects had agreed to such testing, the TB frequency might have been higher.

The culture-based protocol identified a fivefold greater number of TB cases than the older smear-based protocol. TB screening tests, including consecutive sputum MTB culture tests, are essential for the diagnosis of, and epidemiological studies on, TB. The national TB control program needs to be enhanced, including by use of culture tests, if the prevalence of TB in South Korea is to be reduced.

\section{Authors' Contributions}

Conceptualization: Lee S. Methodology: Lee S. Data curation: Ryu JY. Writing - original draft preparation: Lee S. Writing - review and editing: Kim DH. Approval of final manuscript: all 
authors.

\section{Conflicts of Interest}

No potential conflict of interest relevant to this article was reported.

\section{References}

1. World Health Organization. Global tuberculosis report 2015. WHO/HTM/TB/2015.22 [Internet]. Geneva: World Health Organization; 2015 [cited 2017 Apr 1]. Available from: http:// www.who.int/tb/publications/global_report/en.

2. Hong YP, Kim SJ, Lew WJ, Lee EK, Han YC. The seventh nationwide tuberculosis prevalence survey in Korea, 1995. Int J Tuberc Lung Dis 1998;2:27-36.

3. Jeong I, Kim HJ, Kim J, Oh SY, Lee JB, Bai JY, et al. Diagnostic accuracy of notified cases as pulmonary tuberculosis in private sectors of Korea. J Korean Med Sci 2012;27:525-31.

4. Centers for Disease Control and Prevention. Tuberculosis screening and treatment technical instructions (TB TIs) using cultures and directly observed therapy (DOT) for panel physicians [Internet]. Atlanta: Centers for Disease Control and Prevention; 2013 [cited 2017 Apr 1]. Available from: https://www.cdc.gov/immigrantrefugeehealth/exams/ti/ panel/tuberculosis-panel-technical-instructions.html.

5. Diagnostic standards and classification of tuberculosis in adults and children. This official statement of the American Thoracic Society and the Centers for Disease Control and Prevention was adopted by the ATS Board of Directors, July 1999. This statement was endorsed by the Council of the Infectious Disease Society of America, September 1999. Am J Respir Crit Care Med 2000;161(4 Pt 1):1376-95.

6. Lee JY. Tuberculosis infection control in health-care facilities: environmental control and personal protection. Tuberc Respir Dis 2016;79:234-40.

7. World Health Organization. Definitions and reporting framework for tuberculosis: 2013 revision [Internet]. Geneva: World Health Organization; 2013 [cited 2017 Apr 1]. Available from: http://apps.who.int/iris/bitstre am/10665/79199/1/9789241505345_eng.pdf.

8. Korea Centers for Disease Control and Prevention. Annual report on the notified tuberculosis cases patients in Korea 2016. Cheongju: Korea Centers for Disease Control and Prevention; 2017.

9. Liu Y, Painter JA, Posey DL, Cain KP, Weinberg MS, Maloney $\mathrm{SA}$, et al. Estimating the impact of newly arrived foreignborn persons on tuberculosis in the United States. PLoS One 2012;7:e32158.

10. Alami NN, Yuen CM, Miramontes R, Pratt R, Price SF, Navin TR, et al. Trends in tuberculosis: United States, 2013. MMWR
Morb Mortal Wkly Rep 2014;63:229-33.

11. Maloney SA, Fielding KL, Laserson KF, Jones W, Nguyen TN, Dang QA, et al. Assessing the performance of overseas tuberculosis screening programs: a study among US-bound immigrants in Vietnam. Arch Intern Med 2006;166:234-40.

12. Liu Y, Weinberg MS, Ortega LS, Painter JA, Maloney SA. Overseas screening for tuberculosis in U.S.-bound immigrants and refugees. N Engl J Med 2009;360:2406-15.

13. Behr MA, Warren SA, Salamon H, Hopewell PC, Ponce de Leon A, Daley CL, et al. Transmission of Mycobacterium tuberculosis from patients smear-negative for acid-fast bacilli. Lancet 1999;353:444-9.

14. Lewinsohn DM, Leonard MK, LoBue PA, Cohn DL, Daley CL, Desmond E, et al. Official American Thoracic Society/ Infectious Diseases Society of America/Centers for Disease Control and Prevention clinical practice guidelines: diagnosis of tuberculosis in adults and children. Clin Infect Dis 2017;64:e1-33.

15. Hobby GL, Holman AP, Iseman MD, Jones JM. Enumeration of tubercle bacilli in sputum of patients with pulmonary tuberculosis. Antimicrob Agents Chemother 1973;4:94-104.

16. Yeager H Jr, Lacy J, Smith LR, LeMaistre CA. Quantitative studies of mycobacterial populations in sputum and saliva. Am Rev Respir Dis 1967;95:998-1004.

17. Levy H, Feldman C, Sacho H, van der Meulen H, Kallenbach J, Koornhof H. A reevaluation of sputum microscopy and culture in the diagnosis of pulmonary tuberculosis. Chest 1989;95:1193-7.

18. Morgan MA, Horstmeier CD, DeYoung DR, Roberts GD. Comparison of a radiometric method (BACTEC) and conventional culture media for recovery of mycobacteria from smear-negative specimens. J Clin Microbiol 1983;18:384-8.

19. Ichiyama S, Shimokata K, Takeuchi J. Comparative study of a biphasic culture system (Roche MB Check system) with a conventional egg medium for recovery of mycobacteria. Aichi Mycobacteriosis Research Group. Tuber Lung Dis 1993;74:338-41.

20. Alausa KO, Osoba AO, Montefiore D, Sogbetun OA. Laboratory diagnosis of tuberculosis in a developing country 19681975. Afr J Med Med Sci 1977;6:103-8.

21. Liu Y, Posey DL, Cetron MS, Painter JA. Effect of a culturebased screening algorithm on tuberculosis incidence in immigrants and refugees bound for the United States: a population-based cross-sectional study. Ann Intern Med 2015;162:420-8.

22. Posey DL, Naughton MP, Willacy EA, Russell M, Olson CK, Godwin CM, et al. Implementation of new TB screening requirements for U.S.-bound immigrants and refugees, 20072014. MMWR Morb Mortal Wkly Rep 2014;63:234-6.

23. Lowenthal P, Westenhouse J, Moore M, Posey DL, Watt JP, Flood J. Reduced importation of tuberculosis after the implementation of an enhanced pre-immigration screening protocol. Int J Tuberc Lung Dis 2011;15:761-6. 
24. Bai GH, Park YK, Choi YW, Bai JI, Kim HJ, Chang CL, et al. Trend of anti-tuberculosis drug resistance in Korea, 19942004. Int J Tuberc Lung Dis 2007;1 1:571-6.

25. Lee HY, Lee J, Lee YS, Kim MY, Lee HK, Lee YM, et al. Drugresistance pattern of Mycobacterium tuberculosis strains from patients with pulmonary and extrapulmonary tuberculosis during 2006 to 2013 in a Korean tertiary medical center. Korean J Intern Med 2015;30:325-34.

26. Mok JH, Kang BH, Lee T, Lee HK, Jang HJ, Cho YJ, et al. Additional drug resistance patterns among multidrug-resistant tuberculosis patients in Korea: implications for regimen design.
J Korean Med Sci 2017;32:636-41.

27. Vesosky B, Turner J. The influence of age on immunity to infection with Mycobacterium tuberculosis. Immunol Rev 2005;205:229-43.

28. Korea Center for Disease Control and Prevention. Annual report on the notified tuberculosis patients in Korea, 2013. Cheongju: Korea Center for Disease Control and Prevention; 2013.

29. Lee SH. Diagnosis and treatment of latent tuberculosis infection. Tuberc Respir Dis 2015;78:56-63. 